\title{
Therapeutic potential of antiviral drugs targeting chemorefractory colorectal adenocarcinoma cells overexpressing endogenous retroviral elements
}

David Díaz-Carballo ${ }^{1 *}$, Ali Haydar Acikelli', Jacqueline Klein ${ }^{1}$, Holger Jastrow², Philipp Dammann³, Thomas Wyganowski , Cihan Guemues', Sebastian Gustmann', Walter Bardenheuer ${ }^{1}$, Sascha Malak', Nora Sophia Tefett ${ }^{1}$, Veria Khosrawipour ${ }^{4}$, Urs Giger-Pabst ${ }^{4}$, Andrea Tannapfel ${ }^{5}$ and Dirk Strumberg ${ }^{1}$

\begin{abstract}
Background: Endoretroviruses account for circa $8 \%$ of all transposable elements found in the genome of humans and other animals. They represent a genetic footprint of ancestral germ-cell infections of exoviruses that is transmittable to the progeny by Mendelian segregation. Traces of human endogenous retroviruses are physiologically expressed in ovarial, testicular and placental tissues as well as in stem cells. In addition, a number of these fossil viral elements have also been related to carcinogenesis. However, a relation between endoretroviruses expression and chemoresistance has not been reported yet.

Methods: Twenty colorectal carcinoma patient samples were scrutinized for HERV-W $\mathrm{E}_{1}$ and HERV-FRD 1 endoretroviruses using immunohistochemical approaches. In order to search for differential expression of these elements in chemotherapy refractory cells, a resistant HCT8 colon carcinoma subline was developed by serial etoposide exposure. Endoretroviral elements were detected by immunocytochemical staining, $\mathrm{QPCR}$ and ELISA. I $\mathrm{C}_{50}$-values of antiviral and cytostatic drugs in HCT8 cells were determined by MTT proliferation assay. The antivirals-cytostatics interaction was evaluated by the isobologram method.

Results: In this work, we show for the first time that HERV-W $\mathrm{E}_{1}, \mathrm{HERV}-\mathrm{FRD}_{1}, \mathrm{HERV}-\mathrm{3}_{1}$, and HERV-V $\mathrm{V}_{1}$ are a) simultaneously expressed in treatment-naïve colon carcinoma cells and b) upregulated after cytostatic exposure, suggesting that these retroviral elements are intimately related to chemotherapy resistance. We found a number of antiviral drugs to have cytotoxic activity and the ability to force the downregulation of HERV proteins in vitro. We also demonstrate that the use of different antiviral compounds alone or in combination with anticancer agents results in a synergistic antiproliferative effect and downregulation of different endoretroviral elements in highly chemotherapy-resistant colorectal tumor cells.

Conclusions: Enhanced HERV-expression is associated with chemoresistance in colon carcinomas which can be overcome by antiviral drugs alone or in combination with anticancer drugs. Therefore, the introduction of antiviral compounds to the current chemotherapy regimens potentially improves patient outcomes.
\end{abstract}

Keywords: Human endogenous retroviruses, Chemotherapy resistance, Antiviral drugs

\footnotetext{
* Correspondence: david.diaz-carballo@marienhospital-herne.de

'Institute for Molecular Oncology and Experimental Therapeutics, Division of

Oncology and Hematology, Marienhospital Herne, Ruhr University of

Bochum, Medical School, Marienhospital Herne, Duengelstr. 33, 44623 Herne,

Germany

Full list of author information is available at the end of the article
} 


\section{Background}

Cancer chemotherapy is likely to be associated with the development of cancer stem cell-like phenotypes. This chemical stress can force the genesis of cell heterogeneity in the tumor that becomes manifest in its histology, protein expression pattern, and genetic/epigenetic signature [1]. In addition, it is known that drug or ionizing radiation exposure can induce the expression of viral elements present in the cells $[2,3]$. However, the relationship between the manifest endoretroviral spectrum and the development of chemotherapy resistance has not been concatenated until now.

Accounting for $8 \%$ of the human genetic material, human endogenous retroviruses (HERVs) represent a footprint of ancestral germ-cell infections in which viruses integrated into the host genome and were transmitted in a Mendelian form to the progeny [4]. Structurally, HERVs retain all retroviral hallmarks, including the gal, pol \& env genes flanked by non-coding long terminal repeats (LTRs). Although most HERVs have lost the capacity of horizontal transmission due to gene defects, some have retained this ability despite their apparent apathogenicity [5-7]. To ensure proliferation, they sequestrate intact elements from co-expressed exoviruses to form functional entities [8-10].

While organs like ovaries and testes as well as embryonic stem cells express HERV elements abundantly, expression is typically low or non-detectable in somatic cells. Furthermore, it is known that HERV-W significantly contributes to the differentiation of cytotrophoblasts into syncytiotrophoblasts through the fusogenic properties of the syncytins (HERV-W $\mathrm{W}_{\mathrm{E} 1}$ \& HERV-FRD), which are products of the viral envelope gene [11-18].

So far, the contribution of HERVs to normal cell physiology remains largely unstudied. On the other hand, a number of fossil HERVs have been linked to neoplastic transformation that gives rise to breast and small-cell lung carcinomas, renal carcinomas, leukemias, and other malignancies [5, 19-21]. For example, the overexpression of HERV-H and HERV-V-3 was found to be correlated with the development of colon carcinoma, although any relationship to chemotherapy resistance or tumor aggressiveness has not been reported so far $[22,23]$.

It was recently demonstrated that iRNA targeting HERV-K can suppress tumor growth in melanoma models, suggesting that the overexpression of particular HERVs may play a crucial role in tumor physiology [24]. Consequently, interference with these viral elements via antiviral agents could produce antitumoral effects. The introduction of antiviral drugs such as ribavirin into the therapy of tumors with high HERV expression (e.g. refractory AML) has shown complete and partial responses and a reduction in overall levels of eIF4E [25-29]. Nevertheless, the influence of antiviral agents on the expression of these viral elements and their potential anticancer activity has not been reported yet.

Here, we show that cytostatic stress induces the development of highly resistant, HERV-overexpressing tumor cells. We determine the cytotoxic activity of different antiviral agents and highlight their capacity to shut down HERV expression. Finally, we demonstrate that the combination of antiviral compounds and antitumoral drugs reflects synergistic antiproliferative effects in highly resistant, HERV-overexpressing colorectal tumor cells.

\section{Materials and methods}

\section{Cell cultures and patient samples}

HCT8 colon carcinoma cells employed in this study were obtained from the cell and tumor bank of the University of Duisburg-Essen, Medical School. Mononuclear cells (MNC) were isolated from whole blood using Ficoll (Sigma-Aldrich, Missouri, USA) gradient following the manufacturer's instructions. CD34+ cells were isolated using magnetic bead kits (Milteny, Cologne, Germany) following the kit instructions.

\section{Patient samples}

Ethical considerations This study was reviewed and approved by the Committee on Ethics of the Ruhr-University of Bochum, Medical School (register numbers: 4042-11 and 5235-15). Written informed consent was obtained from each participant. Informed written consent regarding eligible subjects below 18 years was obtained from parents. Samples were anonymised, coded and accessible only by research staff. All patient samples were gathered by the division of visceral surgery, Marienhospital Herne, Germany. The histopathological of samples were performed by the institute of pathology of the Ruhr-University of Bochum, Medical School.

\section{$\mathrm{IC}_{50}$ values and induction of etoposide resistance}

$\mathrm{IC}_{50}$ values were determined using the MTT [3-(4,5dimethylthiazol-2-yl)-2,5-diphenyltetrazolium bromide] proliferation assay as described previously, and reported as the mean of three independent experiments. Briefly, cells in exponential growth phase were harvested, washed with medium, and seeded in 96-well plates at appropriate densities according to their growth kinetics. After a conditioning period of 24 hours, cells were exposed to increasing concentrations of cytostatics for 72 hours. The cultures were then incubated with MTT (Sigma-Aldrich, Munich, Germany) dissolved in PBS at a final concentration of $1 \mathrm{mg} / \mathrm{ml}$ for 4 hours. Supernatants were aspirated and the purple formazan crystals dissolved in $100 \mu \mathrm{l}$ of solubilization solution (10 \% SDS in DMSO, Sigma-Aldrich, Munich, Germany). The absorbance was measured in a microtiter plate reader (Infinite 
F200 Tecan, Berlin, Germany) at $570 \mathrm{~nm}$. Both methods were formerly described [30, 31].

Resistance to etoposide in HCT8 cells was induced in the same form previously described $[1,30,32]$. Briefly, $\mathrm{IC}_{50}$ values for cytostatics were determined by MTT assay. Exponentially growing cells were then exposed to $2 \times \mathrm{IC}_{50}$ for 24 hours. For recovery, cells were washed and incubated with drug-free culture medium until new colonies had formed. This procedure was repeated several times, each time doubling the original $\mathrm{IC}_{50}$ until $64 \times \mathrm{IC}_{50}$ was reached. The surviving cells were subjected to a resistance selection by incubation with increasing concentrations of the respective drugs $\left(16 \times\right.$ to $\left.512 \times \mathrm{IC}_{50}\right)$ for 24 hours. Cells which proliferated at higher drug concentrations $(128 \times)$ within one week were considered chemotherapy refractory. Resistant colonies were then expanded in the continuous presence of cytostatics and used for molecular-biological analysis, in particular for studying the expression of CSC features. The resistance factor (RF) was determined by MTT proliferation assay and reported as the $\mathrm{IC}_{50}$ iCSCs/ $\mathrm{IC}_{50}$ parental ratio. Using etoposide as chemoresistanceinducer it is feasible to induce a wide HCT8 subpopulation of cells ( $\mathrm{HCT} 8^{\mathrm{RETO}}$ ) with cancer stem cell features (CSCs) in a very short time. $\mathrm{HCT} 8^{\mathrm{WT} / \mathrm{RETO}}$ cells were cultured in DMEM medium (Biochrom, Berlin, Germany) containing $10 \%$ heat-inactivated fetal calf serum (FCS) and $15 \mu \mathrm{g} / \mathrm{ml}$ Ciprobay (Bayer AG, Wuppertal, Germany).

\section{Studies on the expression of human endogenous retrovirus elements (HERVs) in colorectal carcinomas (CRCs)}

We analyzed the expression of HERV-WE1 and HERVFRD1 in patient samples as well in HCT8 ${ }^{\mathrm{WT} / R E T O}$ colon carcinoma cell line using both immunocytochemical (ICC) and immunohistochemical (IHC) staining.

ICC and IHC staining was performed according to standard protocols [1]. Briefly, ICC cells were grown in chamber slides to appropriate densities, washed with $1 \times$ PBS, fixed with $4 \%$ formaldehyde in PBS for 20 minutes, rinsed twice with $1 \times$ PBS for 5 minutes, and blocked with $10 \%$ normal goat serum (AbD Serotec, London, UK) at room temperature for 60 minutes. For IHC, tissue samples were fixed with $4 \%$ formaldehyde in PBS and embedded in paraffin. Paraffin tissue sections of $4 \mu \mathrm{m}$ thickness were baked overnight at $60{ }^{\circ} \mathrm{C}$ to firmly attach the sections to the slides. After baking, the sections were deparaffinized in 2 changes of xylene-substitute (Thermo Scientific, London, UK) solution for 10-15 min and rehydrated in a series of graded ethanol solutions (100\%, $100 \%, 95 \%, 70 \%, 50 \%)$ for 3 minutes each. HE staining was performed using conventional techniques. For $\mathrm{IHC}$, antigens were retrieved by heating the sections for 30 minutes in $10 \mathrm{mM}$ sodium citrate buffer $\mathrm{pH} 9.0$ at $95{ }^{\circ} \mathrm{C}$ in a domestic vegetable steamer. The slides were washed twice in $1 \times$ PBS for 5 minutes and blocked for 60 minutes with $10 \%$ normal goat serum at room temperature. Primary antibodies (Bioss Antibodies, Woburn, USA and Biorbyt, Cambridge, England) were applied overnight according to the manufacturers' recommendations. On the next day, the slides were washed 3 times in PBST (PBS/0.05 \% Tween 20) for 5 minutes each and rinsed in $1 \times$ PBS for another 5 minutes. Conjugated secondary antibodies (Cell signaling, Cambridge, UK) diluted in PBS/0.05 \% Tween 20/2.5 \% goat serum were incubated for 120 minutes at room temperature according to the manufacturers' recommendations. Next, the samples were stained for 15 minutes with $1 \mu \mathrm{g} / \mathrm{ml}$ Hoechst 33258 diluted in PBS in order to visualize the nuclei. The slides were then washed 3 times in PBST (PBS/0.05\% Tween 20) for 5 minutes each and rinsed in $1 \times$ PBS for another 5 minutes. Tissue specimens were mounted in Faramound Mounting medium (Dako) for visualization.

\section{Differential expression of HERV transcripts in HCT8 ${ }^{\text {WT/RETO }}$ colon carcinoma cells RNA purification and CDNA synthesis}

Total RNA was extracted with Trizol $^{\circledR}$ (Life Technologies, California, USA). To eliminate genomic DNA contamination, the eluted RNA containing 10 IU RNase inhibitor was treated with 7 Kunitz units of RNase-free DNase I (Qiagen, Hilden, Germany) in the appropriate buffer and incubated at $25{ }^{\circ} \mathrm{C}$ for 20 minutes. The RNA samples were then purified further on RNeasy mini columns (Qiagen, Hilden, Germany) according to the manufacturer's instructions. RNA integrity was ascertained by agarose gel electrophoresis and densitometric analysis. $1 \mu \mathrm{g}$ of pure and intact RNA was used for firststrand cDNA synthesis using the cDNA Reverse Transcription Kit from Life Technologies, following the kit instructions.

\section{qPCR}

HERV expression was monitored by qPCR with validated primers and probes from Life Technologies (Cat. Nr.: 18S Hs99999901_s1, HERV WE1 Hs01926764_u1, HERVFRD1 Hs01942443_s1, HERV3-1 Hs 04184598_s1 and HERV-V1 Hs00708335_s1), using the Taqman PCR core reagents according to the manufacturer's recommendations. In addition, the expression of these HERV-elements was confirmed using specific primers purchased from Biomol (Hamburg, Germany). The primers details are reflected in Table 1. The amplification of $25 \mathrm{ng}$ of RNA was performed in triplicate in a CFX96TM Real-Time System (Biorad Laboratories, California, USA). Results were analyzed with CFX-ManagerTM Software Version 3.1 (Biorad Laboratories, California, USA). The evaluation of HERV relative expression was determined using the $\mathrm{Ct}$ comparative method. 
Table 1 Real Time PCR primers used for the detection of HERVs. The accession, region, sequence, polarity and product size for the primers used are reflected

\begin{tabular}{|c|c|c|c|c|c|}
\hline Symbol & Accession & Region & Forward & Reverse & Size $_{(b p)}$ \\
\hline 185 & NR003286 & $1025-1513$ & tcaagaacgaaagtcggagg & ggacatctaagggcatcaca & 488 \\
\hline HERV-W $W_{E 1}$ & AF072506 & $290-463$ & gggttccatggttctcttct & tggtgaaccacttccaagat & 174 \\
\hline HERV-FRD 1 & NM207582 & $504-698$ & ctcattctcacgccttcact & taattccgcctctatgcttg & 195 \\
\hline HERV-V 1 & NM152473 & $1565-1757$ & gggcaaagattctgcaacta & ttgtctggctacctgcctac & 193 \\
\hline HERV-3 1 & NM001007253 & 1377-1562 & taaccagaaattgcctgagc & gaagaggcggttagtgtgaa & 186 \\
\hline
\end{tabular}

\section{Analysis of the simultanean interaction of antiviral and} cytostatic drugs

Amantadine, ribavirin, pleconaril, lamivudine, and doxorubicin were purchased from Sigma-Aldrich, acyclovir and ganciclovir from HEXAL AG, Holzkirchen, Germany. Retrovir was obtained from ViiV Healthcare, London, UK, Foscavir from Clinigen Healthcare, Staffordshire, UK and brivudine from Berlin Chemie, Germany. Etoposide and cisplatin were purchased from TEVA GmbH and 5FU from Medac, both Hamburg, Germany.

The simultaneous effect of antiviral drugs and cytostatics was analyzed by the isobologram method (50\% isodose) as described previously [30]. Briefly, the $\mathrm{IC}_{50}$ for both substances were first determined using the MTT proliferation assay. Applying fixed percentages of the $\mathrm{IC}_{50}$ for the first drug $(20,40,60,80$ and $100 \%)$ and varying the concentration of the second drug from 0.1 to $50 \mu \mathrm{M}$, the variation in the resulting $\mathrm{IC}_{50}$ was determined for every percentage. The same procedure was carried out inversely for the second drug. Dose-response curves were then plotted and evaluated.

\section{Protein isolation and Western blot analysis}

To evaluate the direct effect of antiviral drugs on the expression of HERV proteins we exposure HCT8 cells to amantadine, pleconaril and ribavirin alone or simultaneously at 1 -fold their respective $\mathrm{IC}_{50}$-values for 24 hours. $3 \times 10^{6} \mathrm{HCT}^{\mathrm{WT} / \mathrm{RETO}}$ cells growing exponentially in $75 \mathrm{~cm}^{2}$ TC flasks were incubated in medium containing the respective $\mathrm{IC}_{50}$ of amantadine, pleconaril, and
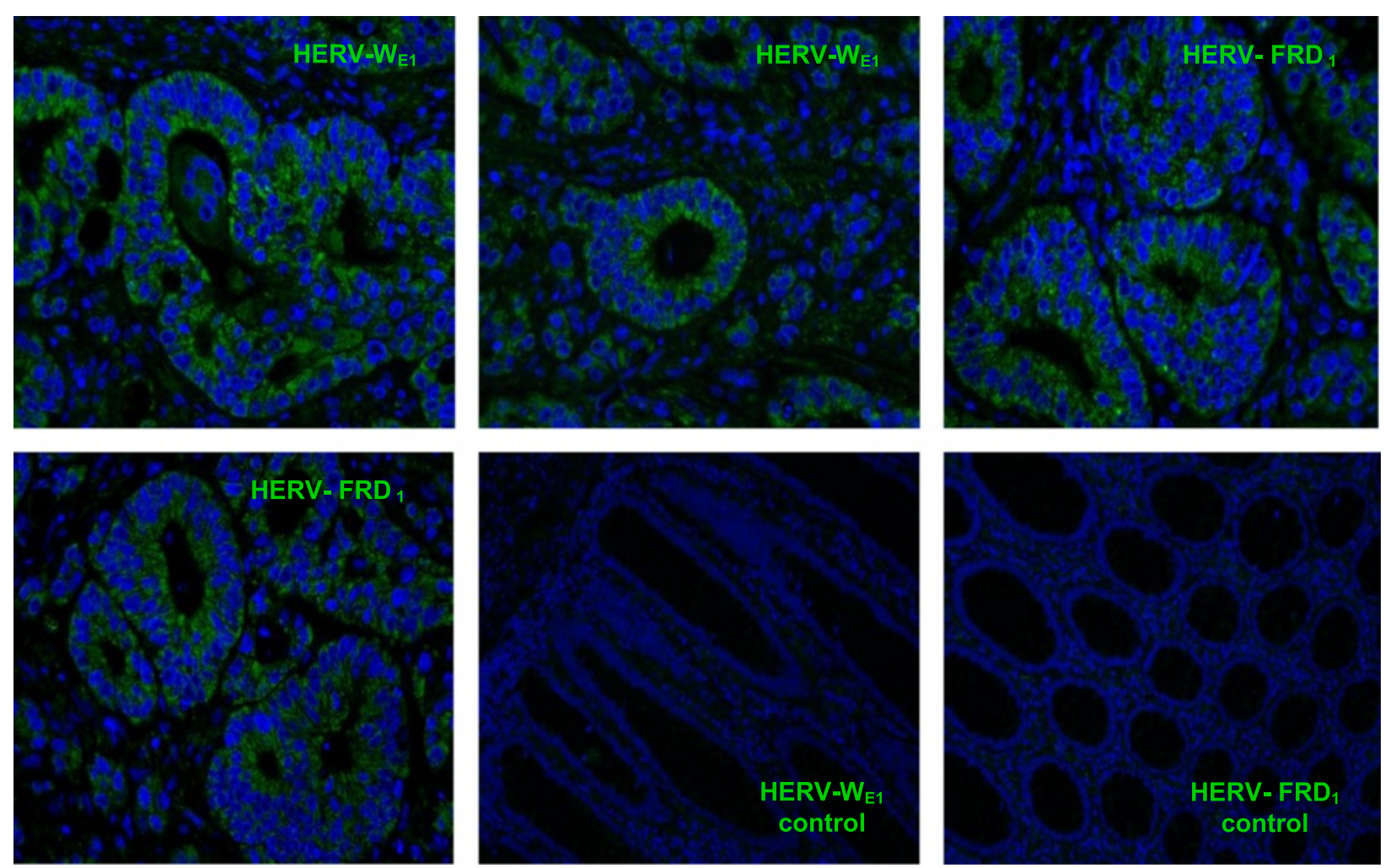

Fig. 1 Overexpression of HERV-W $W_{E 1}$ and HERV-FRD 1 in colon adenocarcinoma patient sections analyzed by IHC. Both elements are principally expressed in the villi, intervillar space and crypts of the large intestine especially in the tumor area, normal tissues are almost negative. Magnification $400 x, n=20$ independent sections 
ribavirin alone or with all drugs simultaneously for 24 hours. Medium was then removed and the cells washed twice with cold PBS. Protein extraction was performed using RIPA buffer as previously described [1]. Briefly, pellets were lysed in RIPA buffer [150 $\mathrm{mM} \mathrm{NaCl}$, $1 \mathrm{mM}$ EDTA, $1 \%$ Triton X-100, 1 \% sodium deoxycholate, $0.1 \%$ SDS, $50 \mathrm{mM}$ Tris- $\mathrm{HCl} \mathrm{pH} \mathrm{7.4]} \mathrm{in} \mathrm{the} \mathrm{pres-}$ ence of a proteinase inhibitor cocktail according to the manufacturer's instructions (Roche Diagnostics $\mathrm{GmbH}$, Mannheim, Germany) for 30 minutes on ice and then centrifuged for 20 minutes at $14000 \mathrm{~g}, 4{ }^{\circ} \mathrm{C}$. The homogenates were measured for protein content using Bradford and normalized to the same protein concentration. Protein extracts $(30 \mu \mathrm{g})$ were resolved by SDSPAGE in a 4-12\% gradient gel (Invitrogen, Karlsruhe, Germany) using Tris-glycine $(0.025 \mathrm{M}$ Tris- $\mathrm{HCl}$, $0.192 \mathrm{M}$ glycine $\mathrm{pH}$ 8.5) buffer, and transferred overnight to $0.2 \mu \mathrm{m}$ nitrocellulose membrane (Pierce Protein, Thermo Scientific Inc., MA, USA). Blots were blocked with $5 \%$ BSA or non-fat milk taking into consideration the recommendations of the manufacturers of the primary and secondary antibodies. Primary antibodies were purchased from Bioss Antibodies, Woburn, USA. Conjugated secondary antibodies were obtained from Cell Signaling and Jackson ImmunoResearch Europe Ltd. (Suffolk, UK). Immunoblots were developed by Western Lightning ${ }^{\bullet}$ Plus-ECL (Perkin Elmer, CA, USA) using a ChemiDoc XRS + system with Image Lab Version 2.0.1 software (Biorad, CA, USA).

\section{Enzyme-linked immunosorbent assay (ELISA)}

Differential HERV expression and its repression by antiviral drugs were monitored using an indirect ELISA method. In brief, 96-well microtiter plates (Greiner Bio-One $\mathrm{GmbH}$, Frickenhausen, Germany) were coated with protein homogenates $(5 \mu \mathrm{g} / 100 \mu \mathrm{l})$ overnight at $4{ }^{\circ} \mathrm{C}$. Well contents were aspirated and the wells washed 3 times with washing buffer (PBS/0.05 \% Tween 20). The wells were then incubated with $300 \mu$ l blocking buffer [PBS/0.05 \% Tween 20/1 \% bovine serum albumin (BSA)] each at $37^{\circ} \mathrm{C}$ for $1 \mathrm{~h}$ and then washed 3 times. Primary antibodies diluted $100 \mu \mathrm{l}$ in blocking buffer 1:500 were added, followed by incubation at $37^{\circ} \mathrm{C}$ for $1 \mathrm{~h}$. The wells were aspirated and washed three times followed by
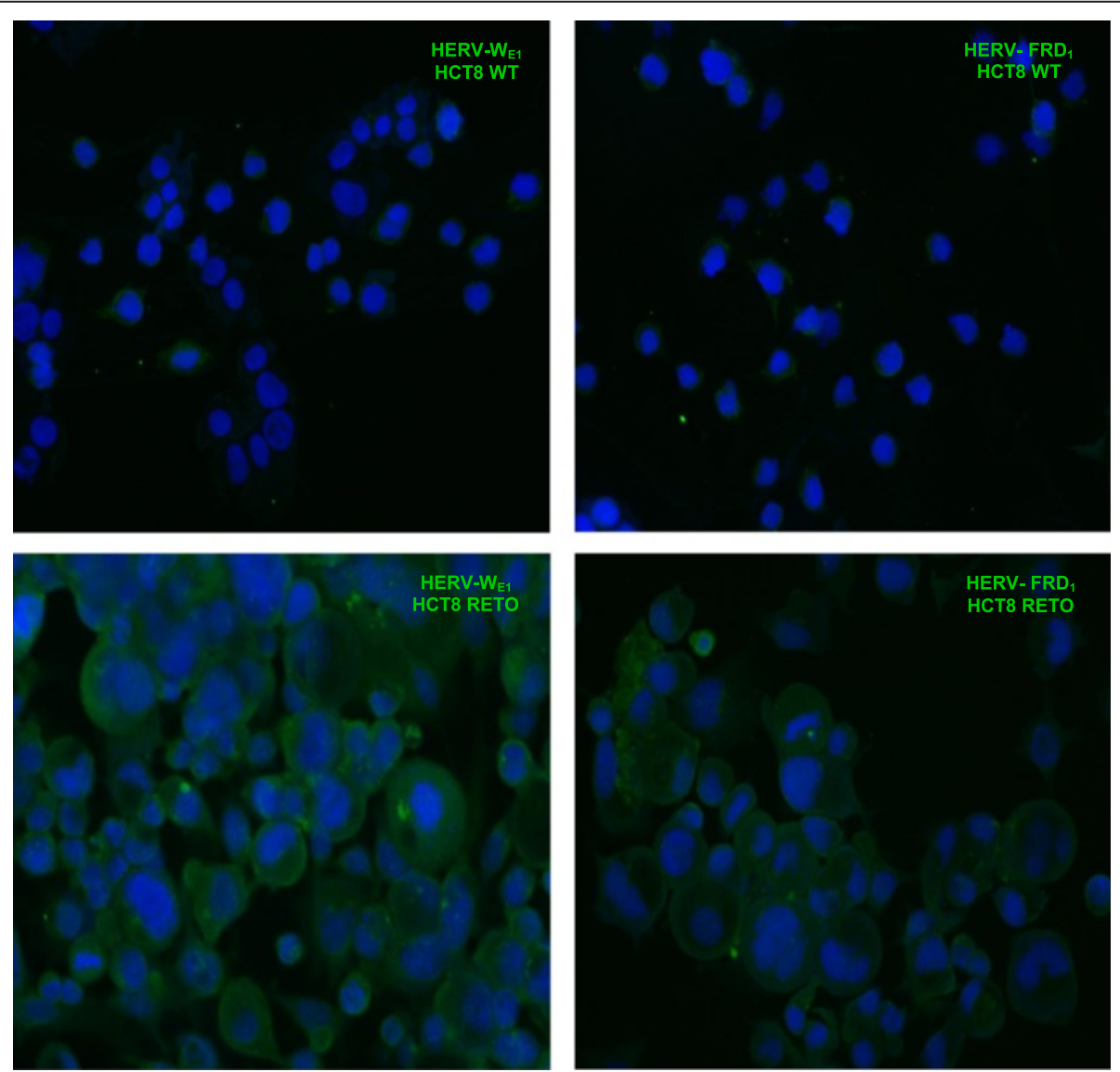

Fig. 2 Differential expression of HERV-W $W_{E 1}$ and HERV-FRD 1 in HCT8 ${ }^{\text {WT/RETO }}$ colon carcinoma cells analyzed by ICC. Cells growing on chamber slides were labeled with anti-HERV-W $\mathrm{E}_{1}$ and anti-HERV-FRD antibodies. Wildtype cells express basal levels of both HERV elements while the HCT8 ${ }^{R E T O}$ cells express high levels and develop hypertrophy. Magnification 400x, $n=3$ independent experiments 
incubation with an HRP-conjugated secondary antibody (Sigma-Aldrich) in $100 \mu \mathrm{l}$ at $37{ }^{\circ} \mathrm{C}$ for $1 \mathrm{~h}$, dilution 1:2000. The wells were washed 3 times and incubated at $37{ }^{\circ} \mathrm{C}$ for $30 \mathrm{~min}$ with $100 \mu \mathrm{l}$ of fresh $0.4 \mathrm{mg} / \mathrm{ml} \mathrm{o}$ phenylenediamine and $0.4 \mathrm{mg} / \mathrm{ml}$ urea $/ \mathrm{H}_{2} \mathrm{O}_{2}$ dissolved in $0.05 \mathrm{M} \mathrm{Na}_{2} \mathrm{HPO}_{4} / 0.05 \mathrm{M}$ citric acid adjusted to $\mathrm{pH}$ 5. The color reaction was stopped with $50 \mu \mathrm{l}$ of $1 \mathrm{M} \mathrm{HCl}$ per well, and the optical density measured after $1 \mathrm{~h}$ at $492 \mathrm{~nm}\left(\mathrm{OD}_{492}\right)$ on an Infinite M200 microtiter plate reader (Tecan, Maennedorf, Switzerland).
Results were normalized using beta-actin as control and presented as percent of expression.

\section{Statistical analysis}

Experiments were performed at least in triplicate and the data given as means \pm standard error of means (SEM), unless stated otherwise. Student's t-test with four degrees of freedom was used to compare independent groups. The statistical analyses were performed with Sigma Plot 12 (Systat Software Inc., California, USA). A probability (p)

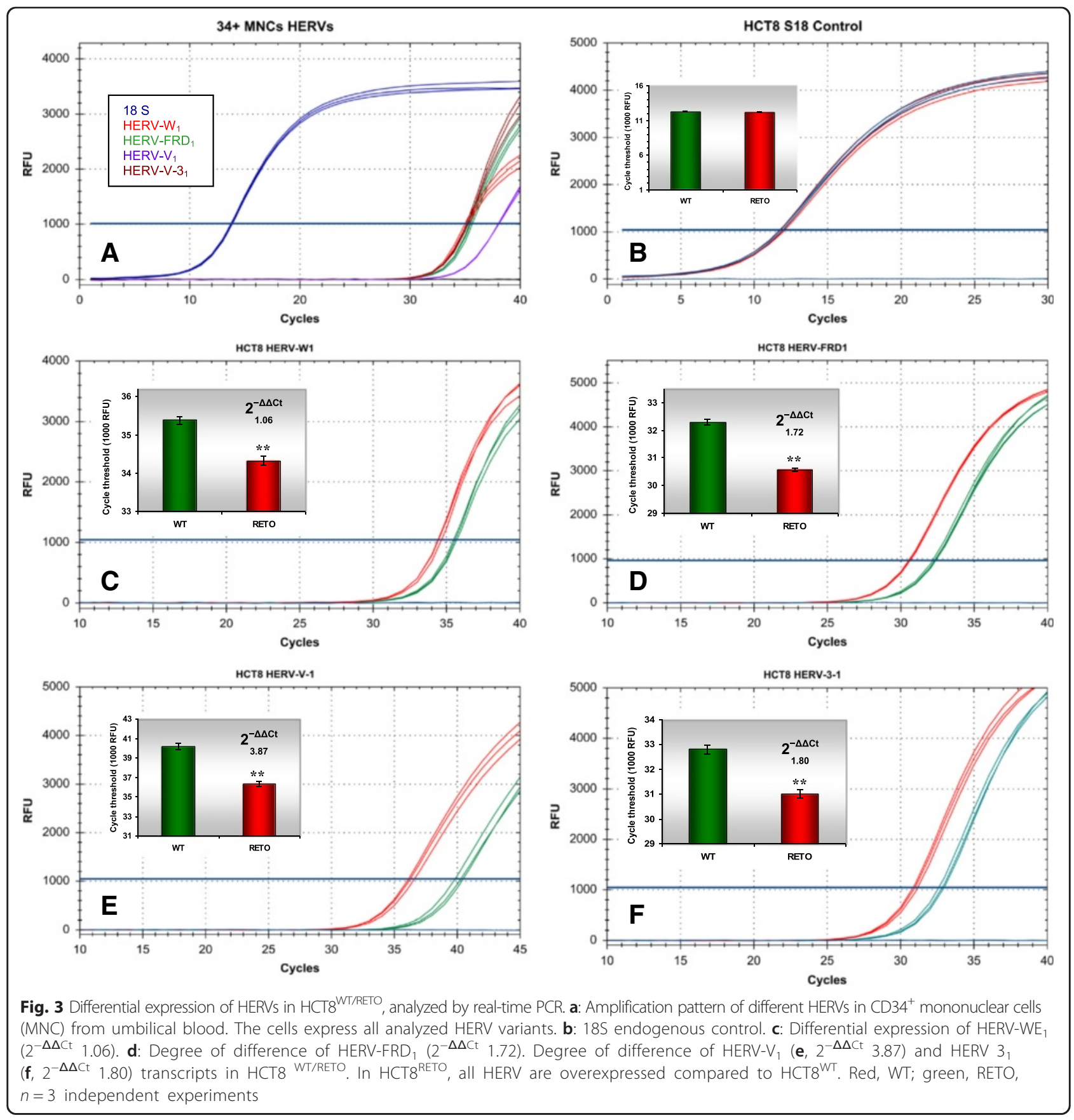


value was considered *: significant $(p<0.05)$; **: very significant $(p<0.01) ;{ }^{* * *}$ : highly significant $(p<0.001)$. ICC and IHC microscopy studies were descriptive and therefore not analyzed statistically; the results shown are representative of at least $n=3$ independent experiments.

\section{Results}

HERV expression in colorectal carcinoma patient material The expression of the HERV elements $\mathrm{W}_{\mathrm{E} 1}$ and FRD was monitored by IHC in human colorectal adenocarcinoma paraffin sections. Both elements were found to be overexpressed in tumors as compared to the normal colon tissues. Fig. 1 shows that the expression of both viral traces was localized to the villi, intervillar regions and the crypts of the large intestine, but confined to the tumor areas.
Overexpression of HERV-W $\mathrm{E}_{1}$ and HERV-FRD 1 in $\mathrm{HCT}^{\mathrm{RETO}}$ colorectal carcinoma cells

The anticancer drug etoposide is commonly not employed in the treatment of colorectal carcinomas (CRC). Nevertheless, this drug is associated with the induction of cancer stem cell characteristics in several tumors as previously described. In HCT8 colorectal carcinoma cells, etoposide induces characteristics for stemness, as judged by their self renewal capacity, colonosphere formation, radioresistance and markedly reduced chemotherapy sensitivity as well as a defined set of CSC-markers like EpCAM, Mucins 1 \& 4, c-Myc, Stat3, stem cell factor (SCF), Musashi, $\beta$-catenin, among others (see Additional file 1). These cells showing CSCfeatures were designated as $\mathrm{HCT} 8^{\mathrm{RETO}}$ cells.

We investigated the differential expression of HERV$\mathrm{W}_{\mathrm{E} 1}$ and HERV-FRD ${ }_{1}$ in colon adenocarcinoma based
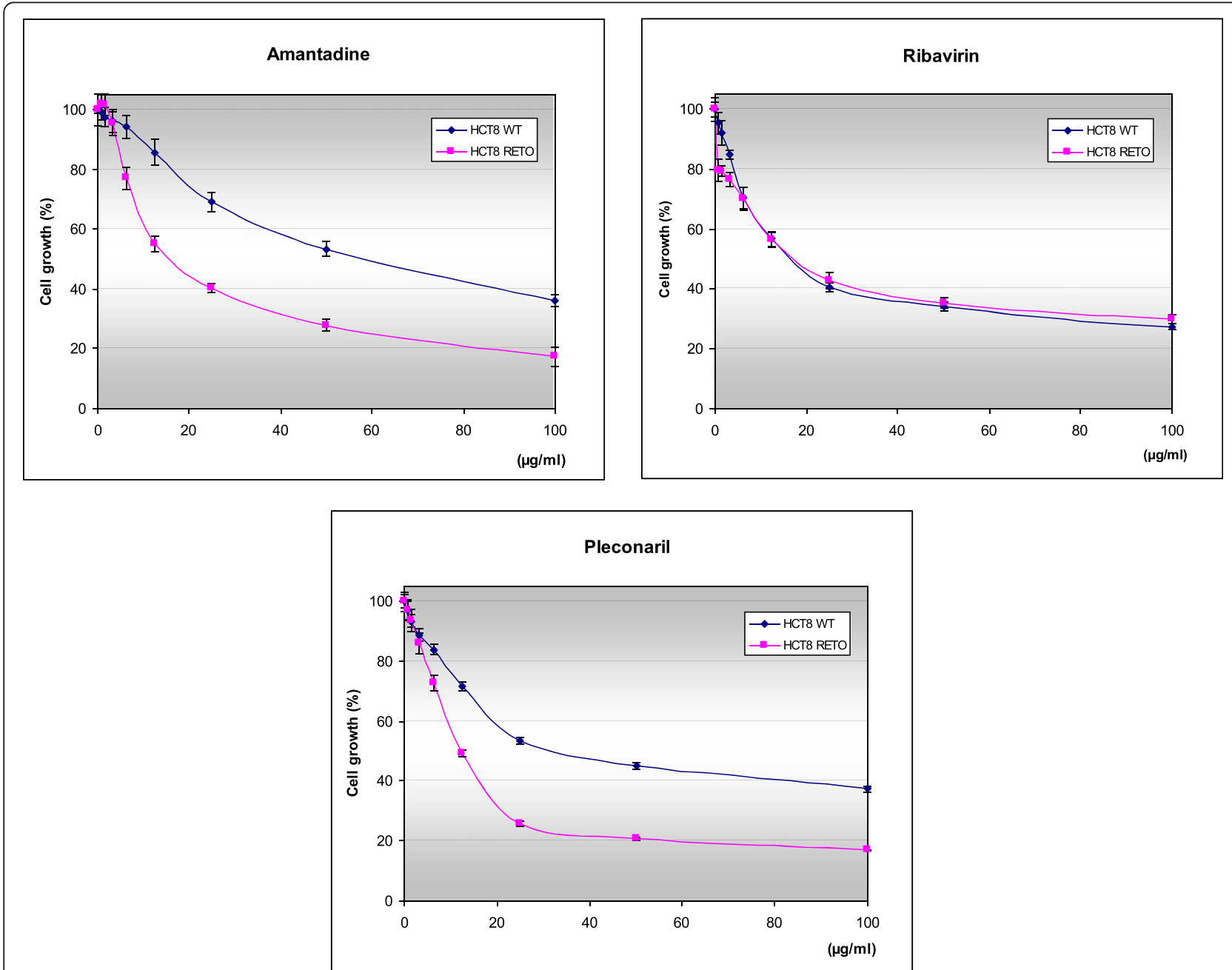

Fig. 4 In vitro cytotoxicity of amantadine, ribavirin and pleconaril in HCT8WT/RETO cells. Cytotoxicity was measured over 24 hours by MTT assay. Amantadine and pleconaril show a specific activity in the highly chemotherapy-resistant subline. Values represent the means \pm standard deviation of at least $n=3$ independent experiments 

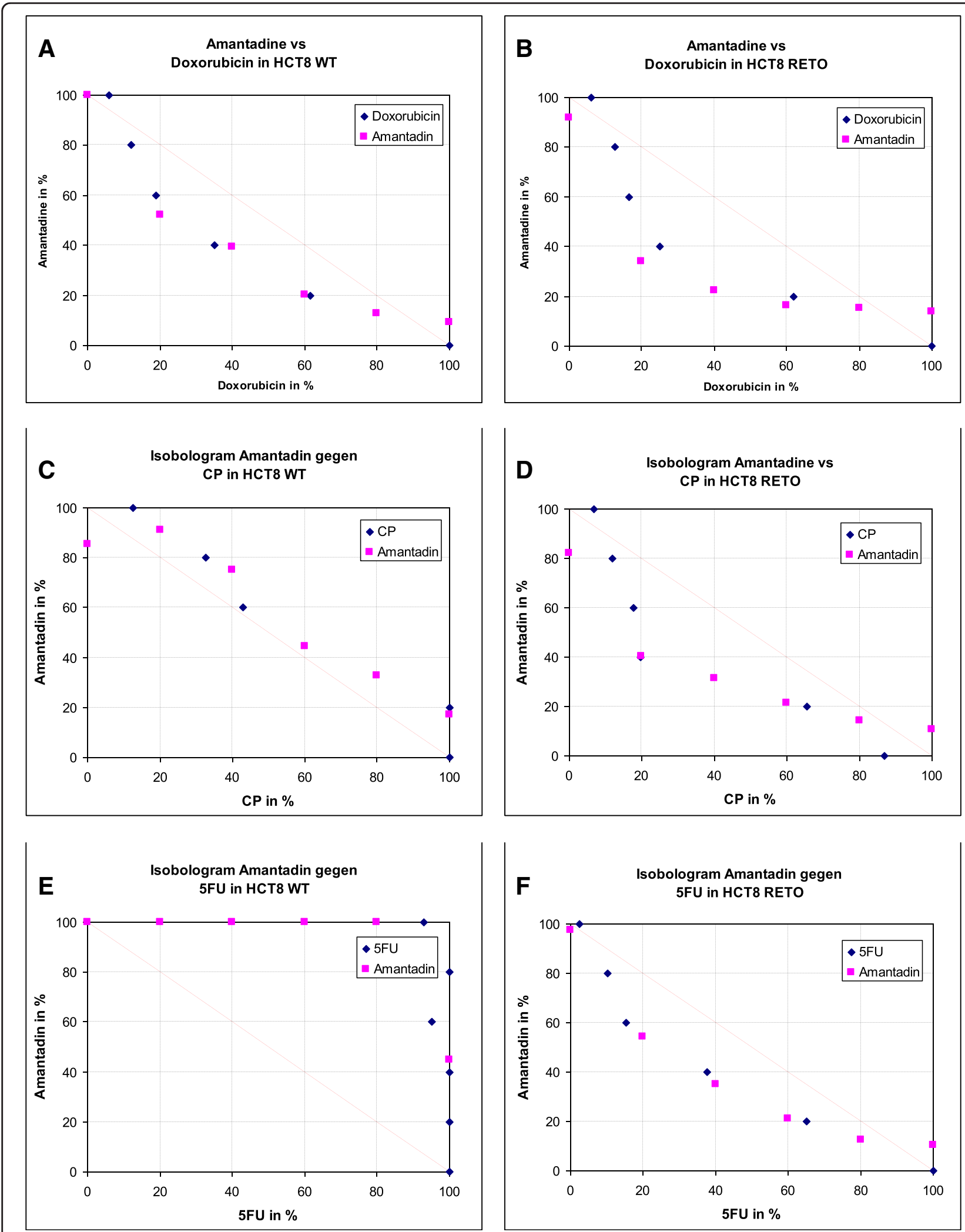

Fig. 5 (See legend on next page.) 
(See figure on previous page.)

Fig. 5 Isobologram showing the in vitro interaction between amantadine and doxorubicin, cisplatin or 5FU in HCT8 $8^{\mathrm{WT}}$ and HCT $8^{\mathrm{RETO}}$ cells.

Dose-response curves are based on the respective $I_{50}$ values calculated after 24 hours of incubation. Amantadine-doxorubicin are synergistic in

HCT8 ${ }^{\text {WT/RETO }}$ (a \& b). Amantadine-cisplatin are additive in HCT8 wildtype (c) and synergistic in HCT8 ${ }^{\text {RETO }}$ (d). Amantadine-5FU are synergistic in

RETO (f) but antagonistic in HCT8 ${ }^{\mathrm{WT}}$ (e). $n=3$ independent experiments

on the cell line HCT8 ${ }^{\mathrm{WT} / \mathrm{RETO}}$. During development of resistance against etoposide, the first observable change was the emergence of a transitory cell hypertrophy as depicted in Fig. 2, together with the overexpression of several endoretroviral elements as observed by ICC.

\section{Various HERVs transcripts are simultaneously expressed in $\mathrm{HCT}_{8}{ }^{\mathrm{WT}}$ and overexpressed in $\mathrm{HCT} 8^{\mathrm{RETO}}$ cells}

Human stem cells have been described to express a range of different HERVs. It is also known that these elements, especially HERV-H, are expressed in colon carcinomas, probably due to the presence of cancer stem cells. The expression of different HERVs and a possible relationship to chemosensitivity has not been reported yet. For this purpose, we analyzed HCT $8^{\text {WT/RETO }}$ cells for HERV mRNAs. Mononuclear CD34 $4^{+}$cells from umbilical cord blood served as positive control since these cells were found to co-express a battery of HERV elements. It was striking, that expression of HERV mRNA transcripts $\mathrm{WE}_{1}$ (Fig. 3c), FRD 1 (Fig. 3d) $33_{1}$ (Fig. 3e) and $\mathrm{V}_{1}$ (Fig. 3f) was significantly enhanced in $\mathrm{HCT} 8^{\mathrm{RETO}}$ cells, compared to parental $\mathrm{HCT} 8^{\mathrm{WT}}$ cells (range from 1.06 to 3.87 -fold).

We next asked whether incubation with antiviral compounds might have an impact on tumor biology and/or proliferation of chemorefractory cancer cells in vitro.

\section{Enhanced cytotoxic effect of antiviral compounds on chemoresistant tumor cells in vitro}

To get a first hint on the antiproliferative activity of antiviral compounds in colon carcinoma cells, we analyzed antiviral drugs like amantadine, ribavirin, pleconaril, lamivudine, acyclovir, ganciclovir, AZT, foscavir and brivudine in HCT8 ${ }^{\text {WT/RETO }}$ cells. Of these, only three compounds showed some degree of antiproliferative effect, as defined by their $\mathrm{IC}_{50}$ values: amantadine, ribavirin and pleconaril (Fig. 4). All other drugs did not show detectable activity at concentrations up to $200 \mu \mathrm{g} / \mathrm{ml}$ (data not shown) and therefore were excluded from further analysis. In general, amantadine and pleconaril were the most active compounds in the HCT8 ${ }^{\text {RETO }}$ cells (Fig. 4).

\section{Synergistic cytotoxic effects between amantadine and anticancer drugs in $\mathrm{HCT} 8^{\text {WT/RETO }}$ cells}

We next questioned the potential interaction between antiviral compounds and classical, clinically used anticancer drugs in vitro. In this work results were only shown for amantadine, but similar data were also achieved for pleconaril and ribavirin, respectively.

In general, using the chemo-sensitive $\mathrm{HCT} 8^{\mathrm{WT}}$ cell line (Fig. 5, panels a, c and e), interaction between amantadine and doxorubicin was slightly synergistic (panel a), between amantadine and cisplatin (CP) additive (panel c), and remarkable antagonistic activity was observed between amantadine and 5FU (panel e), respectively. In contrast, in the chemoresistant HCT8 ${ }^{\text {RETO }}$ cell line (Fig. 5, panels b, d and $\mathrm{f}$ ), synergistic interaction between amantadine and all three anticancer drugs was shown, although synergism was less pronounced using 5-fluorouracil (5FU) (panel f). These results indicate that antiviral compounds like amantadine do not only exhibited substantial cytotoxic activity in chemoresistant tumor cells by itself, but also enhance the cytotoxic efficacy of anticancer drugs in vitro.

We also explored the potential synergistic antiproliferative effects by combining different antiviral compounds by using their specific $\mathrm{IC}_{50}$. As expected, amantadine, pleconaril and ribavirin as single drugs reduced cell survival by about $50 \%$. Enhanced cytotoxicity in $\mathrm{HCT} 8^{\text {RETO }}$ cells was only detectable for amantadine and pleconaril. In general, combination of antiviral compounds (Fig. 6, panel b) resulted in enhanced cytotoxicity, especially for amantadine-containing doublets or even triplets. However, all antiviral combinations did not show enhanced activity in chemo-resistant $\mathrm{HCT} 8^{\mathrm{RETO}}$ cells, compared to chemo-sensitive $\mathrm{HCT} 8^{\mathrm{WT}}$ cells, indicating unspecific cytotoxic effects of antiviral compounds rather than additional suppression of HERV-protein expression.

In addition we analyzed if the antiviral compounds alone or applied as polychemotherapy exerts effects on the regulation of HERVs proteins in HCT ${ }^{\text {RETO }}$ cells, which abundantly expressed different HERVs entities. We found that amantadine, pleconaril and ribavirin alone or in combination applied simultaneously, significantly downregulated the protein expression of HERVs as reflected in Fig. 6, panel b. This effect was drastically observed for the simultanean incubation of all antivirals studied (Fig. 6, panels b \& c). These results certainly demonstrated that cytotoxicity of antiviral compounds in chemoresistant HCT8RETO cells is also associated with downregulation of HERV-proteins.

\section{Downregulation of HERV-proteins upon exposure of chemo-resistant $\mathrm{HCT} 8^{\mathrm{RETO}}$ cells to the antiviral compound amantadine}

We have already shown enhanced cytotoxicity of antiviral compounds in chemoresistant $\mathrm{HCT} 8^{\mathrm{RETO}}$ cells 

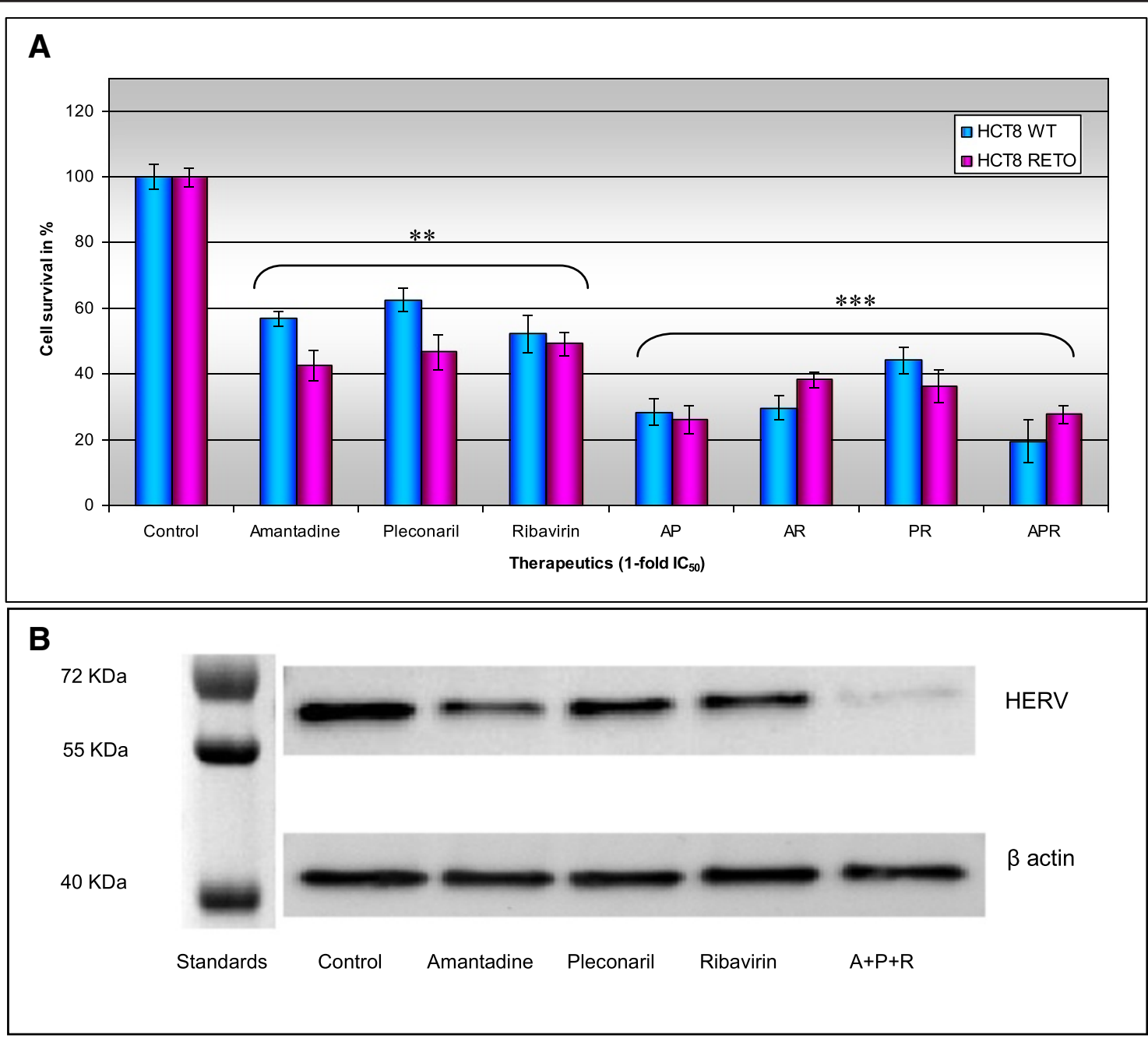

C

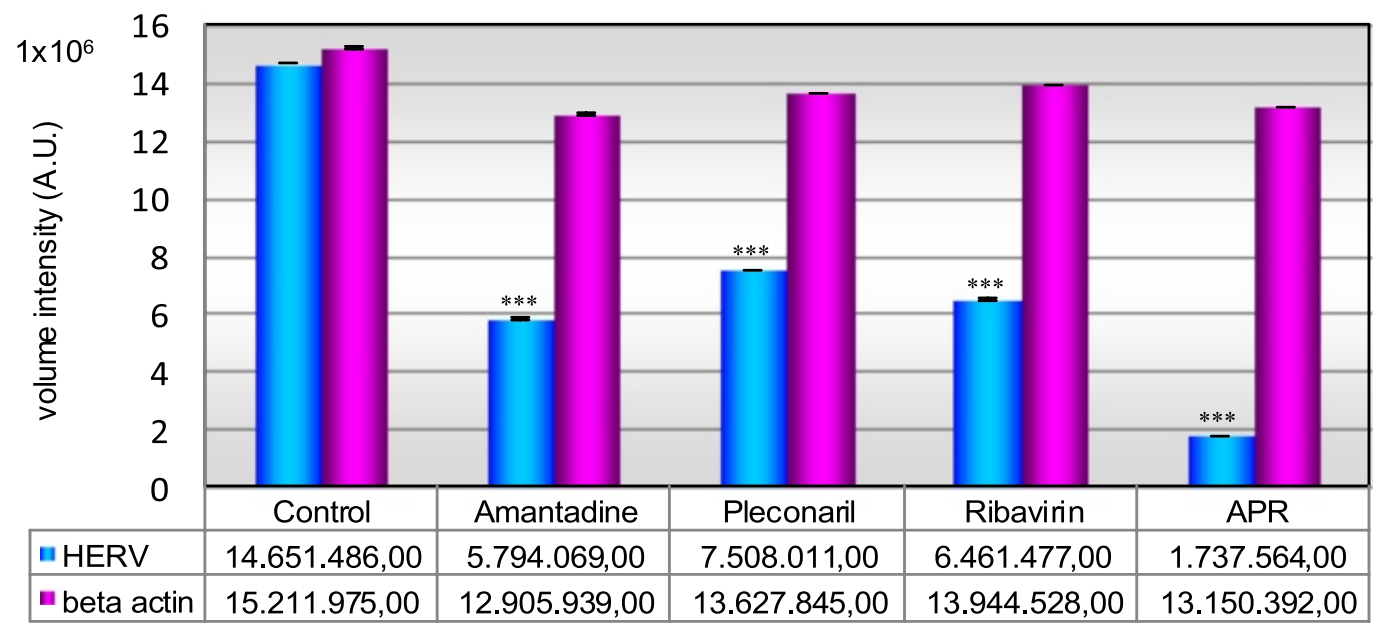

Fig. 6 (See legend on next page.) 
(See figure on previous page.)

Fig. 6 Cytotoxic effect of antiviral drugs and their influence in the expression of HERV proteins in HCT8 colon carcinoma cells. Simultaneous incubation of $\mathrm{HCT}^{\mathrm{WT} / \mathrm{RETO}}$ with amantadine, ribavirin and pleconaril based on the $\mathrm{IC}_{50}$ values calculated for the drugs applied alone or in combination for 24 hours. a: Influence of Amantadine, Pleconaril and Ribavirin in the expression of HERVs analyzed by ELISA in HCT $8^{\text {WT/RETO }}$ cells. b: Down regulation of HERVs proteins after antiviral exposure analyzed by Western blot. c: relative expression of HERVs under the influence of antiviral drugs. Amantadine-pleconaril show a potentiation of circa $35 \%$ in HCT $8^{\mathrm{WT}}$ and $20 \%$ in $\mathrm{HCT}^{\mathrm{RETO}}$. Amantadine-ribavirin have a potentiation of circa $25 \%$ in $\mathrm{HCT}^{\mathrm{WT}}$ and $10 \%$ in HCT8 ${ }^{\mathrm{RETO}}$. The three-way combination ARP was the most effective. Results are representative of $n=3$ independent experiments

in vitro (Fig. 4). Furthermore, we shown that amantadine is able to reduce significantly the HERV protein expression at 1 -fold its $\mathrm{IC}_{50}$ as observed by Western blotting (Fig. 6, panel b \& c). Fig. 7 depicts the semi-quantification of the effect of amantadine in HCT8 ${ }^{\text {WT/RETO }}$ cells as assayed by ELISA. As expected, the protein expression of HERV-W $W_{E 1}$ and $F_{R D}$ is markedly enhanced in $\mathrm{HCT}^{\mathrm{RETO}}$ cells (panel 3) compared to $\mathrm{HCT} 8^{\mathrm{WT}}$ cells (panel 1). Upon incubation with amantadine, pronounced reduction of HERV-W $\mathrm{W}_{\mathrm{E} 1}$ and $-\mathrm{FRD}_{1}$ protein expression was only detectable in $\mathrm{HCT} 8^{\mathrm{RETO}}$ cells. Comparable data were achieved for HERV- $\mathrm{W}_{\mathrm{E} 1}$ and $\mathrm{FRD}_{1}$ at the RNA levels (data not shown).

\section{Discussion}

In this study, we have demonstrated that enhanced expression of various HERV proteins is not only detectable in colon cancer cells, but might also have therapeutic implications for CRC patients especially in chemorefractory tumors.

A number of HERVs have been found to be upregulated during carcinogenesis in tumors derived from tissues that normally show no or only basal expression of these elements. For example, HERV-K transcripts of the Env protein, while entirely absent in normal breast tissue, were demonstrated to be overexpressed in almost all breast carcinomas [33-35]. In addition, the expression of HERV-H and HERV-3-1 in colon carcinomas has been reported [22, 23].

Recently, we described a method to induce multiresistant cancer cells that express several CSC tissuerelated markers as well as stemness features like sphere formation, radio- and chemoresistance [1, 32]. These cells also showed an up-regulation of a set of HERVs. Moreover, HERVs expression has been also linked to stemness in both normal and cancer cells [36].

Colon adenocarcinoma paraffin sections showed significant expression of HERV- $\mathrm{W}_{\mathrm{E} 1}$ and HERV-FRD . These viral transcripts, as well as HERV $3_{1}$ and HERV $V_{1}$, were also expressed in $\mathrm{HCT}^{\mathrm{WT}}$ and overexpressed up to three times in chemotherapy refractory $\mathrm{HCT} 8^{\mathrm{RETO}}$ cells, suggesting a relationship to chemoresistance. Consequently, additional HERV elements might contribute to carcinogenesis and chemotherapy resistance $[4,5,10,11,21]$.

So far, a possible relationship of HERVs with chemotherapy resistance might be a result of the interaction of these proteins with cell membrane structure. Hypothetically, the ability of HERV-W $\mathrm{W}_{\mathrm{E} 1}$ and HERV-FRD proteins to promote cell-cell fusion

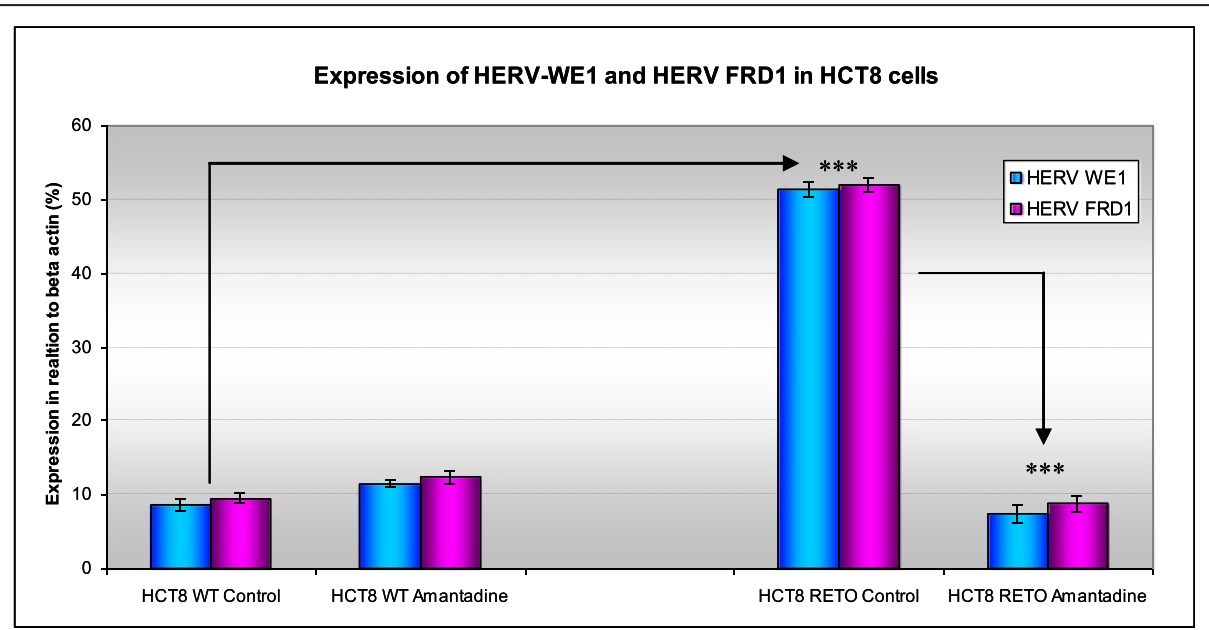

Fig. 7 Effect of amantadine on HERV protein expression in HCT8 ${ }^{W T / R E T O}$ analyzed by ELISA. The expression of both HERV-W ${ }_{E 1}$ and HERV-FRD, is downregulated after incubation with amantadine at $2 \times I_{50}$ for 24 hours. Expression is reduced to basal levels in $\mathrm{HCT}^{\mathrm{RETO}}$. The results are given in percent in relation to the expression of beta-actin as endogenous control 
and generation of multinucleated giant cancer cells could represent an alternative membrane-mediated defense mechanism [12, 14, 15, 37, 38]. Moreover, HERV overexpression could serve as a benchmark to monitor therapy resistance.

The first evidence for the impact of HERV gene repression on the inhibition of tumor cell growth came from the group of Thierry Heidmann [24], who used iRNA directed against HERV-K in a melanoma model. Wang-Johanning and coworker reported on the immunotherapeutic potential of anti-human endogenous retrovirus- $K$ envelope protein antibodies in targeting breast tumors in vitro and in vivo. These anti-HERV-K-specific monoclonal antibodies inhibited tumor growth and induced apoptosis of breast cancer cells [39].

These data served as a rationale to examine antiviral drugs for antiproliferative activity and downregulation of HERV proteins in a panel of HERV-expressing chemoresistant cancer cell lines. Among these compounds, the structurally unrelated amantadine, ribavirin and pleconaril were found to be the most active, with $\mathrm{IC}_{50}$ values below $20 \mu \mathrm{g} / \mathrm{ml}$, that might be clinically relevant.

Amantadine is approved for use as antiviral and antiparkinsonian drug. No primary mechanism of action has been described so far, but it its known that its interference with Influenza virus protein M2 plays an important role in repressing both the early and late phase of viral replication cycle. We focused our studies on amantadine because we previously have been investigating several PPAP compounds (polycyclic polyprenylated acylphloroglucinols) such as nemorosone and plukenetione $\mathrm{A}$, which structurally share the adamantane backbone. We already reported on the antitumoral and antiretroviral activity of these drugs, which we found to inhibit HIV. Moreover, PPAPs were recently described as selective agents in highly resistant neuroblastoma entities [40-43], exerting a pleiotropic effect that involves the downregulation of transcription factors which may interact with viral promoters like Myc, Myb and Stat1/3 [44].

The combination of amantadine with doxorubicin, cisplatin and 5FU acted synergistically in etoposiderefractory $\mathrm{HCT} 8^{\mathrm{RETO}}$, i.e. amantadine boosts the cytotoxicity of these cytostatics in resistant cells.

We also addressed the combination of amantadine, ribavirin and pleconaril on overall cytotoxicity in HCT8 ${ }^{\text {WT/RETO }}$ cells. Enhanced efficacy was observed for the combinations AP (amantadine-pleconaril), AR (amantadine-ribavirin) and ARP (amantadine-ribavirinpleconaril), the latter being the most cytotoxic.

In conclusion, our data suggest that enhanced expression of various HERV proteins might have therapeutic implications in colorectal cancer. Therefore, the introduction of antiviral compounds to the current chemotherapy regimens potentially improves patient outcomes.

\section{Additional file}

Additional file 1: HERV-3 protein downregulation. (DOCX $157 \mathrm{~kb}$ )

\section{Competing interests}

The authors declare that they have no competing interests.

\section{Authors' contribution}

DD-C: Principal investigator. Study conception, design and planning. Biological experiments, data analysis and interpretation. Article conception and preparation. AHA: Biological studies, data acquisition. JK: Biological studies, data acquisition. HJ: Morphological studies on resistant cells. PD: Biological studies, data acquisition. TW: Biological studies, data acquisition. CG: Biological studies, data acquisition. SG: Statistic. Article revision and corrections. WB: Article revision and corrections. SM: Article revision and corrections. NST: Biological studies, article revision and corrections. VK: Surgery, article revision and corrections. UG-P: Surgery, article revision and corrections. AT: Histopathological analysis. DS: Article revision and corrections. Final approval. All authors read and approved the final manuscript.

\section{Acknowledgements}

This investigation was supported by grants afforded by the Marienhospital Herne and the Mildred Scheel Foundation (106993, 108608 Bonn, Germany).

\section{Author details}

${ }^{1}$ Institute for Molecular Oncology and Experimental Therapeutics, Division of Oncology and Hematology, Marienhospital Herne, Ruhr University of Bochum, Medical School, Marienhospital Herne, Duengelstr. 33, 44623 Herne, Germany. ${ }^{2}$ Institute of Anatomy and Experimental Morphology, University of Duisburg-Essen, Medical School, Essen, Germany. ${ }^{3}$ Central Animal Laboratory, University of Duisburg-Essen, Medical School, Essen, Germany. ${ }^{4}$ Department of Visceral Surgery, Marienhospital Herne, Ruhr University of Bochum, Medical School, Herne, Germany. ${ }^{5}$ Institute of Pathology, Ruhr-University of Bochum, Medical School, Bochum, Germany.

Received: 7 April 2015 Accepted: 30 July 2015

Published online: 12 August 2015

\section{References}

1. Díaz-Carballo D, Gustmann S, Jastrow H, Acikelli AH, Dammann P, Klein J, et al. Atypical cell populations associated with acquired resistance to cytostatics and cancer stem cell features: the role of mitochondria in nuclear encapsulation. DNA Cell Biol. 2014;33(11):749-74.

2. Mezhir JJ, Advani SJ, Smith KD, Darga TE, Poon AP, Schmidt H, et al. lonizing radiation activates late herpes simplex virus 1 promoters via the p38 pathway in tumors treated with oncolytic viruses. Cancer Res. 2005;65(20):9479-84.

3. Fonteh $\mathrm{P}$, Meyer $\mathrm{D}$. In vitro reactivation of latent HIV-1 by cytostatic bis(thiosemicarbazonate) gold(III) complexes. BMC Infect Dis. 2014;14(1):680.

4. Stoye JP. Studies of endogenous retroviruses reveal a continuing evolutionary saga. Nat Rev Microbiol. 2012;10(6):395-406.

5. Yu HL, Zhao ZK, Zhu F. The role of human endogenous retroviral long terminal repeat sequences in human cancer (Review). Int J Mol Med. 2013;32(4):755-62.

6. Paces J, Huang YT, Pačes V, Rídl J, Chang CM. New insight into transcription of human endogenous retroviral elements. N Biotechnol. 2013;30(3):314-8.

7. Dewannieux M, Heidmann T. Endogenous retroviruses: acquisition, amplification and taming of genome invaders. Curr Opin Virol. 2013;3(6):646-56.

8. Lee YN, Bieniasz PD. Reconstitution of an infectious human endogenous retrovirus. PLoS Pathog. 2007;3(1):e10.

9. Hanke K, Kramer P, Seeher S, Beimforde N, Kurth R, Bannert N. Reconstitution of the ancestral glycoprotein of human endogenous retrovirus $k$ and modulation of its functional activity by truncation of the cytoplasmic domain. J Virol. 2009:83(24):12790-800.

10. Belshaw R, Pereira V, Katzourakis A, Talbot G, Paces J, Burt A, et al. Long-term reinfection of the human genome by endogenous retroviruses. PNAS. 2004;101(14):4894-9.

11. Seifarth W, Frank O, Zeilfelder U, Spiess B, Greenwood AD, Hehlmann R, et al. Comprehensive analysis of human endogenous retrovirus 
transcriptional activity in human tissues with a retrovirus-specific microarray. J Virol. 2005;79(1):341-52.

12. Lokossou AG, Toudic C, Barbeau B. Implication of human endogenous retrovirus envelope proteins in placental functions. Viruses. 2014;6(11): 4609-27.

13. Kim HS, Ahn K, Kim DS. Quantitative expression of the HERV-W env gene in human tissues. Arch Virol. 2008;153(8):1587-91.

14. Kammerer U, Germeyer A, Stengel S, Kapp M, Denner J. Human endogenous retrovirus $\mathrm{K}$ (HERV-K) is expressed in villous and extravillous cytotrophoblast cells of the human placenta. J Reprod Immunol. 2011;91(1-2):1-8

15. Hayward MD, Pötgens AJ, Drewlo S, Kaufmann P, Rasko JE. Distribution of human endogenous retrovirus type $\mathrm{W}$ receptor in normal human villous placenta. Pathology. 2007;39(4):406-12.

16. Fei C, Atterby C, Edqvist PH, Pontén F, Zhang WW, Larsson E, et al. Detection of the human endogenous retrovirus ERV3-encoded Env-protein in human tissues using antibody-based proteomics. J R Soc Med. 2014;107(1):22-9.

17. Crowell RC, Kiessling AA. Endogenous retrovirus expression in testis and epididymis. Biochem Soc Trans. 2007;35(Pt 3):629-33.

18. Liu Y, Soper C. The Natural History of Retroviruses: Exogenization vs Endogenization. Answers Res J. 2009;2:97-106.

19. Seniuta NB, Kleimman AM, Karseladze Al, Triakin AA, Goncharova EV Tiuliandin SA, et al. HERV-K-associated carcinogenesis: co-expression of viral and cellular proteins in the development of human germ-cell tumors. Vopr Virusol. 2009;54(2):21-6.

20. Kassiotis $\mathrm{G}$. Endogenous retroviruses and the development of cancer. J Immunol. 2014;192(4):1343-9.

21. Downey RF, Sullivan FJ, Wang-Johanning F, Ambs S, Giles FJ, Glynn SA. Human endogenous retrovirus $\mathrm{K}$ and cancer: Innocent bystander or tumorigenic accomplice? Int J Cancer. 2015;137(6):1249-57.

22. Liang $Q, X u Z$, Xu R, Wu L, Zheng S. Expression patterns of non-coding spliced transcripts from human endogenous retrovirus HERV-H elements in colon cancer. PLoS One. 7(1):e29950.

23. Lee SH, Kang YJ, Jo JO, Ock MS, Baek KW, Eo J, et al. Elevation of human ERV3-1 env protein expression in colorectal cancer. J Clin Pathol. 2011;67(9):840-4.

24. Marianne Mangeney JP. Martial Renard, Bertrand Ducos, and Thierry Heidmann, Endogenous Retrovirus Expression Is Required for Murine Melanoma Tumor Growth In vivo. Cancer Res. 2005;65:2588-91.

25. Kraljacic BC, Arguello M, Amri A, Cormack G, Borden K. Inhibition of elF4E with ribavirin cooperates with common chemotherapies in primary acute myeloid leukemia specimens. Leukemia. 2011;25(7):1197-200.

26. Borden KL, Culjkovic-Kraljacic B. Ribavirin as an anti-cancer therapy: acute myeloid leukemia and beyond? Leuk Lymphoma. 2010;51(10):1805-15.

27. Borden $\mathrm{KL}$. Targeting the oncogene elF4E in cancer: From the bench to clinical trials. Clin Invest Med. 2011:34(6):E315.

28. Assouline S, Culjkovic B, Cocolakis E, Rousseau C, Beslu N, Amri A, et al. Molecular targeting of the oncogene elF4E in acute myeloid leukemia (AML): a proof-of-principle clinical trial with ribavirin. Blood. 2009;114(2):257-60.

29. Assouline S, Culjkovic-Kraljacic B, Bergeron J, Caplan S, Cocolakis E, Lambert C. A phase I trial of ribavirin and low-dose cytarabine for the treatment of relapsed and refractory acute myeloid leukemia with elevated elF4E. Haematologica. 2015;100(1):e7-9.

30. Díaz-Carballo D, Malak S, Bardenheuer W, Freistuehler M, Reusch HP. Cytotoxic activity of nemorosone in neuroblastoma cells. J Cell Mol Med. 2008;12(6B):2598-608.

31. Tallarida RJ. An overview of drug combination analysis with isobolograms. J Pharmacol Exp Ther. 2006;319(1):1-7.

32. Díaz-Carballo D, Acikelli AH, Gustmann S, Bardenheuer W, Strumberg D. Acquired resistance to cytostatics triggers cancer stem-cell-like phenotype in different tumor entities. J Stem Cells Regen Med. 2010;6(2):146-7.

33. Wang-Johanning F, Li M, Esteva FJ, Hess KR, Yin B, Rycaj K, et al. Human endogenous retrovirus type $\mathrm{K}$ antibodies and mRNA as serum biomarkers of early-stage breast cancer. Int J Cancer. 2014;134(3):587-95.

34. Wang-Johanning F, Frost AR, Jian B, Epp L, Lu DW, Johanning GL. Quantitation of HERV-K env gene expression and splicing in human breast cancer. Oncogene. 2003;22(10):1528-35.

35. Golan M, Hizi A, Resau JH, Yaal-Hahoshen N, Reichman H, Keydar I, et al. Human endogenous retrovirus (HERV-K) reverse transcriptase as a breast cancer prognostic marker. Neoplasia. 2008;10(6):521-33.
36. Ohnuki M, Tanabe K, Sutou K, Teramoto I, Sawamura Y, Narita M, et al. Dynamic regulation of human endogenous retroviruses mediates factor-induced reprogramming and differentiation potential. PNAS 2014;111(34):12426-31.

37. Rote NS, Chakrabarti S, Stetzer BP. The role of human endogenous retroviruses in trophoblast differentiation and placental development. Placenta. 2004;25(8-9):673-83.

38. Huang G, Li Z, Wan X, Wang Y, Dong J. Human endogenous retroviral K element encodes fusogenic activity in melanoma cells. J Carcinog. 2013;12:5.

39. Wang-Johanning F, Rycaj K, Plummer JB, Li M, Yin B, Frerich K, et al. Immunotherapeutic potential of anti-human endogenous retrovirus- $K$ envelope protein antibodies in targeting breast tumors. J Natl Cancer Inst. 2012;104(3):189-210.

40. Díaz-Carballo D, Ueberla K, Kleff V, Ergun S, Malak S, Freistuehler M, et al. Antiretroviral activity of two polyisoprenylated acylphloroglucinols, 7-epi-nemorosone and plukenetione A, isolated from Caribbean propolis. Int J Clin Pharmacol Ther. 2010;48(10):670-7.

41. Díaz-Carballo D, Malak S, Bardenheuer W, Freistuehler M, Peter Reusch H. The contribution of plukenetione A to the anti-tumoral activity of Cuban propolis. Bioorg Med Chem. 2008;16(22):9635-43.

42. Díaz-Carballo D, Gustmann S, Acikelli AH, Bardenheuer W, Klein J, Dembinski $U$, et al. Multi-targeted polycyclic polyprenylated acylphloroglucinols are major constituents of Cuban propolis and contributors to its anticancer activity. Int J Clin Pharmacol Ther. 2013;51(1):54-5.

43. Díaz-Carballo D, Acikelli AH, Bardenheuer W, Gustmann S, Malak S, Stoll R, et al. Identification of compounds that selectively target highly chemotherapy refractory neuroblastoma cancer stem cells. Int J Clin Pharmacol Ther. 2014;52(9):787-801.

44. Yu H, Liu T, Zhao Z, Chen Y, Zeng J, Liu S, et al. Mutations in 3'-long terminal repeat of HRV-W family in chromomsome 7 upregulate syncytion-1 expression in urothelial cell carcinoma of the bladder through interacting with c-Myb. Oncogene. 2014;33(30):3947-58.

\section{Submit your next manuscript to BioMed Central and take full advantage of:}

- Convenient online submission

- Thorough peer review

- No space constraints or color figure charges

- Immediate publication on acceptance

- Inclusion in PubMed, CAS, Scopus and Google Scholar

- Research which is freely available for redistribution 